\title{
The memristive behavior of non-uniform strained carbon nanotubes
}

\author{
M. V. Il'ina, O. I. Il'in, N. N. Rudyk, A. A. Konshin, O. A. Ageev \\ Southern Federal University, Research and Education Center "Nanotechnologies", Taganrog, Russia \\ mailina@sfedu.ru, ageev@sfedu.ru
}

PACS 73.63.Fg, 77.65.Ly

DOI 10.17586/2220-8054-2018-9-1-76-78

\begin{abstract}
It is shown that the non-uniform elastic strain is the memristive switching origin in carbon nanotubes (CNT). The dependence of the resistance ratio in high- and low-resistance states of the non-uniformly strained CNT on the value strain is obtained. The process of the strain redistribution and its effect on the conductivity of CNT under action of the external electric field strength is studied. The obtained results can be used to develop memristor structures with reproducible parameters based on non-uniformly strained of carbon nanotubes.
\end{abstract}

Keywords: carbon nanotube, strain, memristive switching, piezoelectric effect, scanning tunnel microscopy.

Received: 16 June 2017

\section{Introduction}

The evolution of electronic devices creates a constant demand for the development of new technologies and of operation principles for non-volatile memory. One of the promising development directions in this area is the creation and investigation of memristor structures based on aligned carbon nanotubes (CNT). Previously, we have experimentally shown that vertically aligned CNTs exhibit a memristive switching associated with their strain and polarization [1-3]. Analysis of the literature [4-6] has shown that non-uniform strain in carbon nanostructures can lead to the appearance of a piezoelectric effect and the corresponding internal electric field in them. We put forward a proposal that the non-uniform strain of the CNT acts as an additional source of resistance which depends on the value of the current flowing. The application of an external electric field to the non-uniformly strained nanotube can lead to the redistribution of the strain and a reproducible switch of nanotube resistance.

The aim of the research is to study a memristive behavior of carbon nanotubes at different non-uniform strain values.

\section{Experimental studies}

The experimental sample of the aligned carbon nanotube array was created by plasma-enhanced chemical vapor deposition (PECVD). CNT array images were obtained by scanning electron microscopy (SEM) Nova NanoLab 600 (FEI, Netherlands) and scanning tunneling microscopy (STM) using probe nanolaboratory Ntegra (NT-MDT, Russia) are shown in Fig. 1. The study of the sample by the Raman spectrometer Renishaw InVia Reflex (Renishaw plc, UK) showed the presence of D-, G-, and $\mathrm{G}^{\prime}$-modes, which is typical for multi-walled aligned carbon nanotubes [7].

The memristive behavior of the individual strained CNT with diameter $(D)$ of $92 \mathrm{~nm}$ and length $(L)$ of $2.2 \mu \mathrm{m}$ was investigated by the STM in the current spectroscopy mode at applying a sawtooth voltage pulse with amplitude $U$ from \pm 1 to $\pm 10 \mathrm{~V}$. The localization of the STM probe over the individual nanotube top was carried out after the scanning of the CNT array in the constant current mode of STM (Fig. 1b). The upper electrode was a tungsten probe with a radius of $146 \mathrm{~nm}$. The lower electrode was a conducting layer on the substrate surface. The non-uniform elastic strain $\Delta L(x)$ in the nanotube was created under the action of an external electric field before the measuring the current-voltage characteristics (CVCs). The strain value $\Delta L(L)$ from 0.2 to $3.0 \mathrm{~nm}$ was controlled by the STM feedback system. The surface potential of strained nanotubes was obtained by the Kelvin Probe method (nanolaboratory Ntegra probe).

\section{Results and discussion}

The dependence of resistance ratio in high- and low-resistance states $\left(R_{H R} / R_{L R}\right)$ of the individual strained CNT ( $D=92 \mathrm{~nm}, L=2.2 \mu \mathrm{m})$ on the strain value is shown in Fig. 2. This dependence was obtained based on the CVCs of the CNT at $U=8 \mathrm{~V}$ and the reading voltage at $1 \mathrm{~V}$. It was found that the maximum value of the $R_{H R} / R_{L R}$ corresponded to the $\Delta L(L)=1.2 \mathrm{~nm}$, a decrease or increase in this value $\Delta L(L)$ led to decrease in the ratio $R_{H R} / R_{L R}$ (Fig. 2). This was due to the fact that strain $\Delta L(L)=1.2 \mathrm{~nm}$ corresponds to the condition of the compensation of the initial internal electric field of the non-uniform strained CNT by the piezoelectric field 

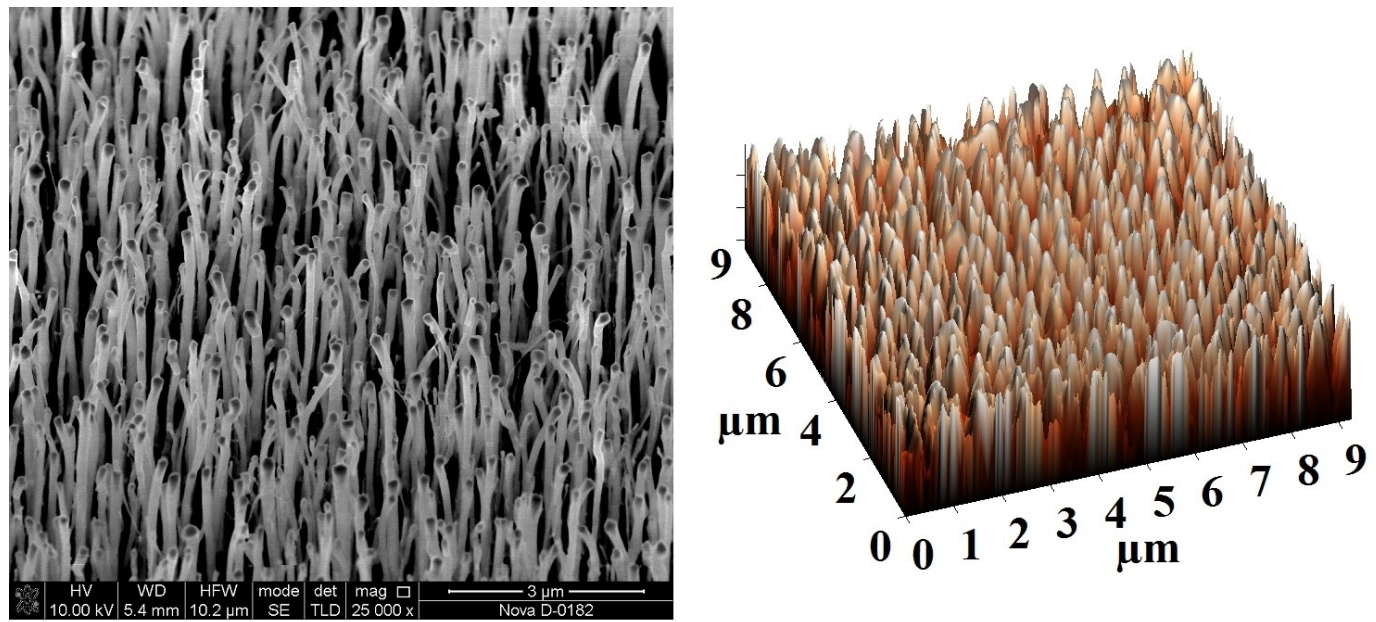

FIG. 1. The experimental sample of the CNT array: SEM image (a) and STM image (b)

that occurred at applying an external electric field. The resistance of the CNT having small strain $(>0.5 \mathrm{~nm})$ changes insignificant $\left(\sim 48 \mathrm{M} \Omega, R_{H R} / R_{L R} \approx 1\right)$ at applying an external electric field due to low value of the internal electric field of the nanotube. Increasing the strain $\Delta L(L)$ to $3 \mathrm{~nm}$ leads to the fact that the value of the arising piezoelectric field becomes insufficient to compensate substantially increased internal electric field in the strained CNT. As a result, the resistance of the strained CNT increases to $252 \mathrm{M} \Omega$ and the ratio $R_{H R} / R_{L R} \approx 1$ (Fig. 2).

The results for investigation of the strain redistribution process and its effect on the conductivity of CNT at $\Delta L(L)=1.0 \mathrm{~nm}$ are shown in Fig. 3 .

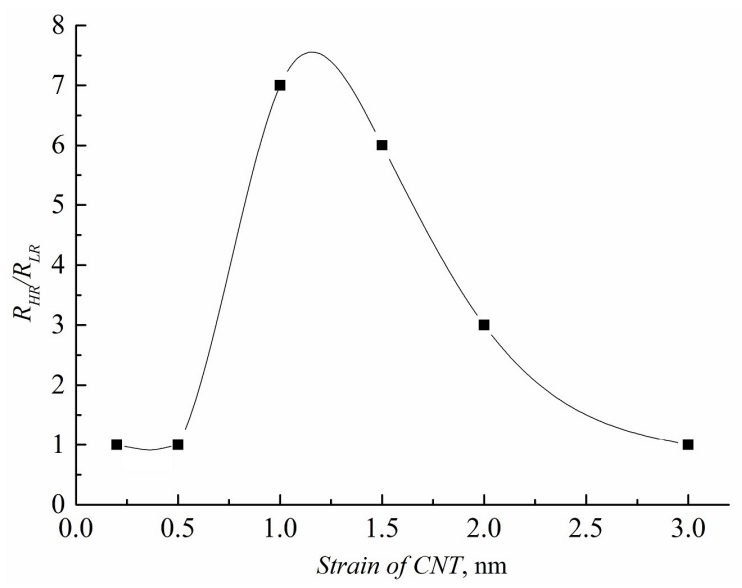

FIG. 2. The dependence of CNT $R_{H R} / R_{L R}$ on the strain value at $U=8 \mathrm{~V}$

Analysis of the obtained CVCs of the strained CNT (Fig. 3) showed that at low voltage pulse amplitude values ( $U \leq 4 \mathrm{~V}$ ), the ratio $R_{H R} / R_{L R}$ was also close to 1 . This is due to the insufficient external electric field strength value for the strain redistribution in the nanotube under the action of a piezoelectric effect. A further increase in the voltage pulse amplitude led to an increase in the external electric field strength and the gradual redistribution of the CNT strain and a change in its internal electric field. As a result, the ratio $R_{H R} / R_{L R}$ began to increase with increasing the amplitude value.

The study of the strained carbon nanotubes by the Kelvin probe method confirmed on their tops the presence of a positive surface potential from 3 to $78 \mathrm{mV}$ depending on the magnitude of the tension. The cause of surface potentials at the nanotube tops may be the non-uniform elastic strain and piezoelectric effect [6]. 


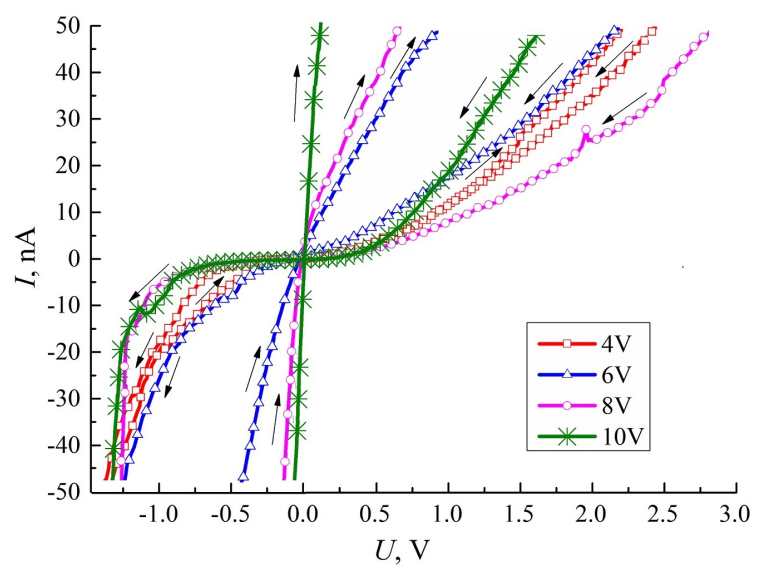

FIG. 3. The CVCs of the nanotube with $\Delta L=1.0 \mathrm{~nm}$ at different value of the $U$

\section{Conclusion}

Thus, the strain in the nanotube acts as a source of additional resistance and the redistribution of this strain caused by an external electric field leads to a change of nanotube resistance and the emergence of current-voltage curve hysteresis. The resistance ratios in high- and low-resistance states of the non-uniformly strained CNT are determined by strain values and the external electric field strength.

The conducted researches are directed to development of the interdisciplinary scientific direction - straintronics, which studies the change in the physical properties of nanostructures due to elastic strain arising under the action of controlled external influences. The results obtained can be used to develop promising elements of nanosystems and nanoelectronics based on non-uniformly strained carbon nanotubes, in particular, memristor structures with reproducible parameters.

\section{Acknowledgements}

The results were obtained using the equipment of Research and Education Center and the Center for Collective Use "Nanotechnologies" of Southern Federal University.

This work was supported by the Russian Foundation for Basic Research (project No. 16-29-14023 ofi_m) and by Southern Federal University (grant No. VnGr-07/2017-26).

\section{References}

[1] Ageev O.A., Blinov Yu.F., Il'in O.I., Kolomiitsev A.S., Konoplev B.G., Rubashkina M.V., Smirnov V.A., Fedotov A.A. Memristor Effect on Bundles of Vertically Aligned Carbon Nanotubes Tested by Scanning Tunnel Microscopy. Tech. Phys., 2013, 58(12), P. 1831-1836.

[2] Ageev O.A., Blinov Yu.F., Il'in O.I., Konoplev B.G., Rubashkina M.V., Smirnov V.A., Fedotov A.A. Study of the Resistive Switching of Vertically Aligned Carbon Nanotubes by Scanning Tunneling Microscopy. Phys. Solid State, 2015, 57(4), P. 825-831.

[3] Ageev O.A., Blinov Yu.F., Ilina M.V., Ilin O.I., Smirnov V.A. Modeling and experimental study of resistive switching in vertically aligned carbon nanotubes. J. Phys. Conf. Ser., 2016, 741(1), P. 012168.

[4] Zelisko M.A., Hanlumyuang Y.B., Yang S.C., Liu Y.D., Lei C.D., Li J.D., Ajayan P.M., Sharma P. Anomalous piezoelectricity in two-dimensional graphene nitride nanosheets. Nat. Commun., 2014, 5, P. 4284.

[5] Chandratre S., Sharma P. Coaxing graphene to be piezoelectric. Appl. Phys. Lett., 2012, 100, P. 023114.

[6] Wang X., Tian H., Xie W., Shu Y., Mi W.-T., Mohammad M.A., Xie Q.-Y., Yang Y., Xu J.-B., Ren T.-L. Observation of a giant two-dimensional band-piezoelectric effect on biaxial-strained grapheme. NPG Asia Materials, 2015, 7, P. e154.

[7] Antunes E.F., Lobo A.O., Corat E.J., Trava-Airoldi V.J. Influence of diameter in the Raman spectra of aligned multi-walled carbon nanotubes. Carbon, 2007, 45, P. 913-921. 